\title{
Development and Implementation of the Veterans Administration's Multihospital Radiology Information System
}

\author{
Morris L. Gavant
}

\begin{abstract}
Unknown to most radiology professionals, the Veterans Administration (VA) is implementing an automated radiology information system as an integrated component of its Decentralized Hospital Computer Program. The basic design has been evaluated and refined over the past 5 years. It is now becoming available in all 172 VA medical facilities. Radiology services are provided in a complex management and fiscal environment. The primary purpose of the information system is to improve the efficient processing, performance, and reporting of requests for radiologic consultations and procedures. The automatic capturing of demographic and medical statistics will provide local and national managers more complete data with which to plan future financial, equipment, and personnel requirements. The VA radiology module has the potential to influence the shape of all future systems, commercial and public. This report describes the development of this radiology information system, its current status, and its potential impact on the largest health care system in the country. The module serves as an example of what can or should be expected from the radiology portion of a comprehensive medical information management system.
\end{abstract}

(C) 1989 by W.B. Saunders Company.

COMPUTER-BASED RADIOLOGY information systems (RIS) are slowly replacing the established labor intensive data management systems in hospitals, clinics, and private radiology practices. ${ }^{1-4}$ The most publicized systems are commercial endeavors by private industry that have been developed in cooperation with universities or other institutions with large radiology facilities.

Unknown to most of the country's radiologists, a comprehensive, integrated RIS is currently being developed and implemented by the Veterans Administration (VA) via the radiology portion of its Decentralized Hospital Computer Program (DHCP). ${ }^{5,6}$ Several factors suggest that this system may ultimately influence the shape of future radiology information systems.

Because this RIS will be placed in the largest medical system in the United States, many radiologists and other physicians in their capacity as VA staff physicians, as academic and consulting physicians, and as residents-in-training will be exposed to this system as it is fully installed over the next 5 years. This report presents a brief history of the DHCP, and describes the development and purpose of the radiology special-interest user group (SIUG), the module's current status, and its future course and shape.

\section{Background}

The VA supervises the largest health care system in the country. 1.3 million veteran patients are hospitalized and more than 20 million outpatient visits are made each year. Obviously, data collection, management, and analysis with a federal government agency of this size is not simple. The DHCP was implemented in 1982 to automate the administrative, personnel, and health care services in all VA medical centers.

The first phase, implemented in 172 facilities, includes the "core" modules, supporting medical administration, pharmacy, and clinical laboratory services. This phase included patient data base development via the automation of registration, admission, discharge, and transfer, and outpatient scheduling information. This data base of patient information is the foundation of the entire system from which the other modules are built and designed. The next step beyond this basic system is known as the enhanced core and includes four additional areas ("Core +4 ") radiology, dietetics, medical records tracking, and accounting and procurement capabilities.

The radiology module will replace independent local VA medical centers' efforts of varying sizes and complexities with a standard system. Many of the best functions and options in these preceding efforts are intentionally incorporated into the VA software to maximize the ease of day-to-day use of the system. The standardization of reporting and management information will provide local and national planners uniform,

From the Departments of Radiology, Memphis Veterans Administration Medical Center and the University of Tennessee-Memphis.

Address reprint requests to Morris L. Gavant, MD, Department of Radiology, University of Tennessee-Memphis, 800 Madison Ave, Memphis, TN 38163.

(c) 1989 by W.B. Saunders Company.

0897-1889/89/0203-0002\$03.00/0 
objective data, which can assist in determining future financial allocations for labor, equipment, and building requirements.

This complex software program, developed by federally employed programmers using taxpayers' dollars, is available in the public domain. It will be and has already been used by private developers in their commercial products. For instance, the Department of Defense (DOD), through its Defense Medical Systems Support Center (DMSSC), recently awarded a $\$ 1$ billion contract for their Composite Health Care System (CHCS) to the San Diego-based Science Applications International Corporation. ${ }^{5}$ This firm will install a patient data management system based on the VA's DHCP using the Massachusetts General Hospital's Utility MultiProgramming System (MUMPS) and Digital Equipment Corporation (DEC; Maynard, MA) hardware for the DOD's 172 military hospitals and 587 military medical facilities worldwide. In addition, the Indian Health Service has also decided to use this software to automate its medical data management system. ${ }^{5}$

\section{Hardware}

DHCP hardware currently consists of DEC PDP 11/44 and 11/84 central processing units (CPU) with various peripheral terminals and printers. Special interfaces are also used with clinical laboratory devices. Recently awarded federal contracts call for DEC to upgrade the CPUs in larger facilities to the VAX 6220 or 6230 models or MicroVAX systems of multiple 3500 or 3600 machines. Smaller facilities will then receive the $11 / 44$ and $11 / 84$ as hardware upgrades. ${ }^{6,7}$

\section{MUMPS}

The DHCP and the separate modules which compose it are unique in several ways. All DHCP applications are developed using MUMPS, which was originally developed as an interpreted computer language in the 1960s and received American National Standards Institute approval in 1977. The language is available in compiled versions. Its "string" manipulation capabilities and its inverted "B-tree" structure allows for automatic alphabetized sorting of data. Thus, it integrates the system support software with a standard patient database.
The VA Kernel is the software link between the vendor's machine and the DHCP operating system. The Kernel link gives the VA the independence and flexibility to select the vendor hardware most appropriate for the needs of its facilities, which have varying automation needs. The FileManager, a part of the Kernel, is the DHCP's database manager. It can handle large changing files as data-base software does. The Kernel also allows each application to be installed in a modular fashion. In addition, it supports menus, files, data security, on-line assistance/help, and electronic-mail functions. ${ }^{8,9} \mathrm{Ad}$ hoc queries and reports are also available through the FileManager.

\section{Radiology Special-Interest User Group}

A prototype development approach was used in designing and writing the software. A specialinterest user group (SIUG) was organized. It was composed of the clinical users from VA facilities around the country and the software developers. By corresponding in person, via conference calls, or by electronic mail, the users described to the program developers directly and in nontechnical terms their clinical needs and the capabilities they required from the software. The programmers wrote the software, and the SIUG reviewed the results, providing comments and criticism. Through a reiterative process, the software module was carefully refined to meet the perceived current needs of the prospective user. This ongoing approach provides a product responsive to improvements in technology or changes in management requirements. Obsolesence is unlikely.

The VA has a vast system of 172 hospitals, each of which theoretically operates with the same management, budgeting, and financial system. Nevertheless, variations in data collection, patient care, and management do develop, even within a rigid and bureaucratic government system. Therefore, the menu-driven program is designed with flexibility in mind. Each facility is allowed multiple start-up and daily operation options, which can customize much of the software to be used in a given facility.

\section{Developer Support}

The software developers are supported by seven regional information system centers (ISC) 
that are responsible for DHCP installation and maintenance as well as program and troubleshooting support. The ISC in Troy, NY has been responsible for the software development of the radiology module.

\section{Current Status of Radiology Software}

The SIUG approved the basic form of the radiology module software in 1985 . The $\alpha$ or initial test site for the program was at the Albany, NY VA Medical Center. After this initial phase to detect unforeseen problems, implementation was expanded to $\beta$ sites. This was the last step for refinement and revision prior to general release of the program to all ISC and VA facilities. The released software was then made available to all VA medical facilities that had proper radiological and computer personnel and hardware support. Currently, it has been implemented in 91 VA medical centers.

The functional components of the software are as follows: Ward/Clinic Order Entry; Exam Entry/Edit; Films Reporting; Management Reports; Outside Film Registry; Patient Profile; Radiology Module Supervisor; and User Utilities.

A menu-driven program guides the user through the system with prompts at all information entry points. This allows for rapid training of personnel who may have had minimal computer or keyboard experience. Because interactions with the system vary with work responsibilities, standard or customizable menus are available for clerical, technical, medical, and supervisory personnel within the imaging department as well as for referring health care providers and physicians.

Ward/clinic order entry. Clerical and nursing personnel electronically transmit requests for radiologic procedures via the radiology order entry program. Depending on the complexity of the request history, the experience of the user, and the processing speed of the main computer, each request takes approximately 1 minute to enter. The on-line request with the pertinent patient and clinical information is then immediately printed at the designated location within the radiology department, ie, computed tomography section, outpatient department, etc. This "working" document follows the examination through the department. It is discarded once the examination results are transcribed into the computer.

Computer-based order entry eliminates delays in the scheduling and performing of procedures caused by problems with the writing and delivery of manually created requests. For instance, prior to the introduction of automated order entry at our facility, conventional delivery of consultation requests through interhospital mail or pneumatic system took from several hours to several days and was fraught with misplaced and lost requests. The need for these delivery methods has been eliminated.

The automated printing of the pertinent clinical history, patient identification and location data, and the name of the requesting physician has eliminated delays due to illegible or improperly written requests. The automated process has also provided previously unavailable data regarding the exact time a request is sent to and received by the radiology department. This process has reduced interdepartmental conflicts caused by misplacement or nondelivery of consultation or procedure requests.

Each medical center must decide whether or not to provide its physicians access to the electronic radiology order entry. Medicolegally, each facility may want to ensure that a written order is properly placed into the medical record and limit physician access to the order entry system.

With the on-line availability of all pending requests, simple computer-based procedure scheduling abilities are available. These can replace current labor intensive or nonexistent scheduling systems. Examinations can be scheduled by date and time. This information is available on line at any hospital terminal and allows more efficient coordination among the various nursing and ancillary services for planning multiple radiologic and nonradiologic diagnostic and therapeutic procedures. More refined techniques allowing scheduling by individual examination room, procedure, and technologist work load require further development.

Exam entry/edit. When a patient is registered for a procedure, a custom-designed flash card and a film jacket label are automatically printed. A unique case number is assigned each procedure. This number will remain associated 
with the examination until a verified report is entered into the computer.

A list of recently performed studies is displayed. Those recently performed with barium or water-soluble contrast agents are annotated with warning messages to prevent the improper timing of procedures.

After the examination, the radiology technologist performing the procedure identifies him- or herself as having performed the study and confirms that the procedure requested has been performed. Alternatively, the technologist may edit the procedure to indicate cancellations, changes, or additional studies. In addition, specific film usage data (customized to size, brand, or even company name) may also be entered. This editing process takes the technologist approximately 1 minute per procedure. The information is used to provide personnel workload, examination room workload, and film usage reports.

Examination reporting. The radiology physician interprets and dictates the full consultation. Using a standard word processing program imported from the VA's DHCP, transcriptionists enter the draft report at computer work stations. The typical automated document editing functions and the on-line availability of demographic, clinical, and procedure-related data improves the transcriptionists' efficiency and productivity. Typewriters are eliminated. Batched or individual reports are printed, reviewed, and signed by the radiologist. As a last step, the verification of the report is noted in the computer.

At the department's option, the report draft may be made available in a released but unverified form as soon as it is transcribed into the computer. It is then immediately available on line to any health care provider with proper computer access at any terminal in the hospital. Unverified drafts are corrected as necessary. Once verified, the report can be corrected by adding an amendment.

The on-line availability of radiology procedure findings eliminates delays and frustrations caused by an incomplete or an unavailable paper-based medical record. Excluding the time required to send and match the printed request with the medical record, on-line reports are available for review from one to three days earlier than the written report.

In the past, clerical personnel at our facility typically spent at least 4 to 6 hours a day trying to discover if procedures had been performed and interpreted and, if so, if a typed report was available. At least several radiology staff-hours a day were wasted repeating interpretations and dictations because of lost or misfiled reports or examinations that had not been dictated. With the ability to determine if on-line reports are available, these costly and inefficient uses of personnel were replaced with more productive employment.

The report body and impression are kept available together online as long as possible. If the computer's memory approaches capacity, the report body usually may be purged for those studies more than 1 year old. An off-line taped back-up of the entire report is always maintained.

Management reports. Multiple management reports are available not only for internal department use but also for local, regional, and national planning and budgeting decision making. Daily internal department reports include listings of delinquent or nondictated procedures, abnormal exams, and complications. Daily logs of procedures performed, patient preregistration lists, and lists of outside films requiring return are also readily available.

Work-load reports track specific details regarding type and volume of procedures performed within the department. Procedures are automatically coded and weighted by work units ( $a$ form of relative value scale) in a format acceptable for reporting to the central VA budgeting and planning staff via its automated management information system (AMIS) as well as by alternative methods that allow comparison of VA institutions with non-VA medical and radiological facilities. Reports are also available to track examination room use, staff or resident radiologist work load itemized by procedure, individual and overall radiological technologist workload, and referring physician/clinic/bed-section volume.

Outside film registry. An automated registry is available to track the films received from outside sources. Reports can be triggered daily to provide lists of films that need to be returned. The registry can flag these films to require confirmation prior to their return.

Radiology module supervisor. Various startup tasks and maintenance routines are provided 
for the supervisory personnel managing the radiology module software. The routines in installing the software package to establish site-specific parameters include (1) defining requirements for placement of the patient or their films into various examination statuses. These are used to track patients, uninterpreted films, or unverified reports which may be in the department. Statuses might include separate categories for patients waiting for an examination, those already examined, those procedures transcribed but unverified, and those procedures which are verified and completed; (2) specification of individual registration locations within the department, ie, main desk, computed tomography, outpatient department; (3) designing templates for flash cards, film jacket labels, and radiology reports; and (4) examination room descriptions.

Maintenance functions include (1) report and examination deletion; (2) updating or adding to the list of active radiology procedures; (3) updating radiology personnel employment status and individual accessibility to the system; and (4) entering descriptions of film types used within the department.

Patient profile. A profile listing all the radiology examinations performed on a patient is available at any computer station in the hospital. This routinely provides such information as the referring physician's name, the patient's location, the current status of an examination, the technologist who performed the study, and the radiologist who supervised or interpreted the examination. Times of status change are available as activity and status tracking logs. Thus, objective data on when a patient was registered and examined and the time from the performance of the procedure to the on-line availability of the radiology report was easily determined.

User utilities. Functions are available to enter information regarding patients; this will be displayed at registration. This includes notification of prior contrast reactions and identifying patients who may, for a variety of reasons, have difficulty cooperating with the performance of a radiographic procedure.

\section{Future Status}

Several refinements of the system software are currently undergoing preliminary field testing. These functions include on-line physician verifi- cation of radiology reports, bar-code tracking of radiology film jackets, and sophisticated schedul ing functions. As these upgrades are released, their integration into a given medical center's radiological information system will primarily depend on CPU capacity, input/output device availability, and computer systems personnel support.

On-line physician verification of procedure reports. This software enhancement will allow on-line review of the draft examination report with verification via electronic physician signatures. Radiologists will access the report verification menu at regular intervals. They will then confirm their unique electronic signatures and review and "sign" all the procedure reports transcribed in their names. Alternatively, they may verify reports of other physicians who are unable to review their own studies. This will automatically associate or print the verifying physician's verification signature with the on-line or printed report.

Once the radiology report has been verified, it can be immediately transmitted to the originating outpatient clinic or to the current inpatient location for printing and placement into the medical record. If desired, a report can be printed in the radiology department for filing with the patient's film jacket.

Thus, on-line verification will decrease the delays in returning reports because of patient transfers within or discharge from the hospital or because of illegible location data on the handwritten request. Delays caused by manual or mechanical delivery systems can also be eliminated.

The electronic signature function has necessitated the development of a policy at the national and local level on computer security and confidentiality of electronically stored patient and physician identification data. Individual employees must acknowledge their legal and medical responsibilities to secure password and signature codes that could provide unauthorized access to confidential patient and physician user information.

Bar-code tracking of radiology film jackets. Bar-code identification labels and bar-code readers to track supplies and the locations of medical and radiographic records has become quite familiar in many businesses and medical data management systems. A bar-code method for tracking film jackets is currently undergoing $\beta$ testing. 
This system will maintain information on the locations of all radiographic film jackets removed from the department for physician review. In addition, intradepartmental localization of film jackets is also possible. Potentially, the exact location of the film jacket by viewing station or examination room within the radiology department is possible.

This capability will eliminate the time-consuming need for radiology personnel to search for missing or misplaced film jackets within the department or in wards and clinics. Automated check-out logs will maintain lists of the individual or clinic responsible for the given film jacket if it is removed from the department. Overdue messages will be available to prevent personnel from removing additional film jackets from the department until delinquent jackets checked out in their names have been returned.

\section{Impact on Patient Care}

As with most computer systems used in clinical medicine, the radiology module of the DHCP will improve the care and the administrative functioning of the hospital and the radiology department because of its ability to provide rapid transmission of and access to medical and demographic patient information. Delays in transmission of such data as requests and reports will be eliminated. This can decrease the time between placement of the request to the return of a verified report to the medical record by several days. This will allow radiology personnel to more efficiently perform their assigned duties because time-consuming tasks such as finding missing or misplaced requests, reports, and film jackets will be eliminated. Given the number of facilities involved, the number of work hours saved may be immense.

Clerical delays in receiving or processing requests or consultations for radiological procedures will no longer prolong patient hospitalization or potentially adversely affect patient treatment or outcome. Patient film jackets removed from the radiology department for physician review will be locatable prior to the performance of surgical procedures. In addition, accurate accounting of procedures and radiology staff work loads will allow for improvements in identifying and planning for local, regional, and national needs within the VA's system.
The automated radiology module will allow for a more accurate determination of current personnel workload and procedure volume. No procedure will be performed or credited without accessing the radiology information system. In addition, studies removed or misplaced prior to official interpretation by the radiologist can be identified and located. This improvement in data collection will assist local, regional, and national health planners in determining or defending resource allocation decisions.

As each facility implements the radiology information package, naturally there will be resistance to change from the familiar manual system. The initial confusion will pass as personnel learn to interact with the computers and their software. Extensive in-service training for clerks, nurses, and physicians would be ideal. However, the funds to provide training personnel and equipment may be limited. A great advantage of the MUMPS and DHCP softwares are their ease of use. The time required for entering radiology request data is minimized because much of the information is already available in the main patient data base. Self-explanatory prompts are used to enter all other information. Differences in information management methods among the various radiology departments in the VA system will occur. The ability to customize many of the software functions will minimize these differences.

Finally, although the federal government has provided the funding support for the development of a rather extensive and elegant software, budget limitations may limit the availability of hardware. Thus, the radiology information system may only function as a complete package in larger facilities with an adequate CPU capacity and a supply of input/output devices. The advantage of the current software is that it can be implemented in steps and grow as the hardware abilities of the given facility increase.

\section{ACKNOWLEDGMENT}

Acknowledgment must be given to the many men and women who contributed to the development of the radiology module through their past and current involvement as system programmers, as radiologist participants in the special interest user group, and as general and VA policy makers and health planners. Current members of this team include Jim Demetriades, Robin Ostrander, Don Marsett, Linda Weiss, Dan Bishop, and Bob Eaton, MD. 


\section{REFERENCES}

1. Shannon RH: Information integration for the imaging specialist. Radiol Clin North Am 24:121-130, 1986

2. Bauman RA: Radiology information systems, in Taveras JM, Ferrucci JT (eds): Radiology Diagnosis-ImagingIntervention (chapter 34) Philadelphia, Lippincott, 1987, pp $1-6$

3. Jost RG: Radiology reporting. Radiol Clin North Am 24:19-26, 1986

4. Jost RG: Monitoring department efficiency and quality. Radiol Clin North Am 24:27-37, 1986

5. Cox DA (ed): VA computing. Issue 2. An information bulletin for VA computer users. Washington DC: Veterans
Administration's Office of Information Systems and Telecommunications, End User Assistance Service, 1988, pp 3-9

6. Vizachero R: Strong bases of computing power. Gov Comput News 10:4-5, 1988

7. Pietrucha B: VA hospital system awaits upgrade. Gov Comput News 10:10, 16, 1988

8. Walters RF, Bowie J, Wilcox JC: MUMPS Primer: An Introduction to the Interactive Programming System of the Future, (ed 2). College Park, MD, 1982 MUMPS User Group, 1983, pp 1-12

9. Smith JY, Harvey WJ: MUMPS is germinating business productivity. J Systems Management 5:26-31, 1988 